\title{
Pursuit-Evasion Game for Normal Distributions
}

\author{
Chanyoung $\operatorname{Jun}^{\dagger}$
}

\author{
Subhrajit Bhattacharya ${ }^{\dagger}$
}

Robert Ghrist ${ }^{\dagger}$

\begin{abstract}
In this paper we consider a probabilistic model of a pursuit-evasion game on $\mathbb{R}^{n}$, in which neither pursuer nor evader positions are known with certainty. Both parties are represented by normal distributions that evolve according to a Kalman filter as new sensor readings (observation from overhead camera/satellite images) are obtained. The objective is to design the control commands issued by the pursuer (which is executed noisily). The control commands issued by the evader are unknown - only sensor measurements are given. Even with such limited knowledge we prove boundedness of a distance between the pursuer's distribution and the evader's true distribution (one that takes into account the evader's control commands). Our simulation results support the claimed guarantees.
\end{abstract}

\section{INTRODUCTION}

Consider the pursuit-evasion game played by two agents on $\mathbb{R}^{n}$ - an evader, $E$, moving in the space, and a pursuer, $P$, trying to catch it [4], [2], [8]. Typically the future motion of the evader is not known (or only some bounds on velocity or a coarse strategy is known), and the task at hand is to design a control strategy for moving the pursuer so that it eventually catches the evader. The precise definition of 'catch' may vary. Often, given the unpredictability of the evader, the desired objective is to be able to reach the evader within a finite distance.

In this paper we consider a discrete-time single-pursuer $(P)$ single-evader $(E)$ game on $\mathbb{R}^{n}$, but instead of representing the agents as points in $\mathbb{R}^{n}$, we represent them by normal distributions on $\mathbb{R}^{n}$. Such requirements may arise when, for example, the precise location of the agents are not known, but only approximate/noisy sensor measurements are available. This, at best, allows us to infer a probability distribution about the location of the agents. In particular, we will model the pursuer and the evader as normal distributions that evolve according to the rules of a Kalman filter [3], updated by noisy sensor observations. The goal is to design control velocities for the pursuer so as to catch the evader. The support of the [normal] distributions being the entire $\mathbb{R}^{n}$, one cannot use a simple Euclidian metric to measure the distance between the agents. Instead we employ a metric on distributions to compare $E$ and $P$.

Probabilistic pursuit-evasion games such as this have been studied, mostly in general probability distribution settings [10], [5]. Although more general in their setup, such algorithms are accompanied by little-to-no theoretical guarantees. In this paper, we aim for a stronger theoretical

Department of Mathematics, University of Pennsylvania, Philadelphia PA 19104. e-mail: cyjun2@gmail.com, subhrabh@math. upenn.edu, ghristemath.upenn.edu. The authors acknowledge the support of federal contracts FA9550-12-10416 and FA9550-09-1-0643. foundation to this line of research. In particular, we propose some weak assumptions and design control strategies for the pursuer such that the distance between their probability distributions remain bounded by a finite positive value: a no-win criterion for the evader. The reason for an emphasis on (pseudo-) metrics on distributions (as opposed to the simpler mean-pursuit approach [10]) is to prepare for a theory adapted to multimodal distributions.

\section{A. Problem Description}

We represent agents, $P$ and $E$, in Euclidean $\mathbb{R}^{n}$ as nondegenerate normal distributions, $\phi^{P}$ and $\phi^{E}$ respectively, which are the best estimates for their true distribution. A Kalman filter can be used to update the distributions as sensor readings about the positions of the respective agents (e.g., readings from overhead/satellite camera) are obtained. A Kalman filter can also incorporate information about noisy velocity commands (control inputs/commands) issued by the respective agents to compute even better estimate of the distributions.

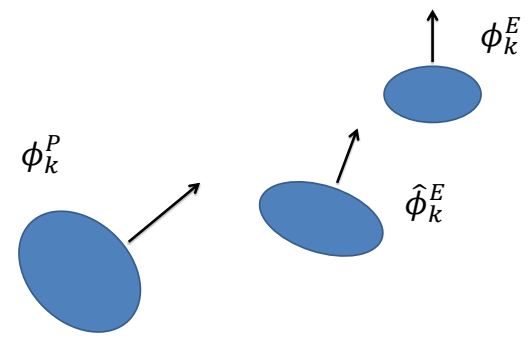

Fig. 1. The true evader $\phi_{k}^{E}$, the estimated evader $\hat{\phi}_{k}^{E}$ and the pursuer $\phi_{k}^{P}$

Assume that sensors obtain noisy position readings of both the agents, and that the sensor model is known, and that the sensor can communicate with the pursuer, $P$, to convey the readings. The pursuer also knows the control command that it issues at every [discrete] time step. However we do not assume that the control commands issued by the evader are known. Hence, in our problem, we have the following three distributions:

i. $\phi^{P}$ : This is the normal distribution representing the pursuer, $P$, and is updated using a Kalman filter as new sensor readings of the position of the pursuer $\left(\left\{z_{0}^{P}, z_{1}^{P}, z_{2}^{P}, \cdots\right\}\right)$ and the control commands issued by the pursuer $\left(\left\{d_{0}^{P}, d_{1}^{P}, d_{2}^{P}, \cdots\right\}\right)$ are obtained. This distribution is of course at the disposal of the controller for designing the control commands for the pursuer.

ii. $\hat{\phi}^{E}$ : This is the estimate of the normal distribution representing the evader, $E$, and is updated using a 
Kalman filter as new sensor readings of the position of the evader $\left(\left\{z_{0}^{E}, z_{1}^{E}, z_{2}^{E}, \cdots\right\}\right)$ arrive. However the update cannot use the control commands issued by the evader, since that information is unavailable to the pursuer. This distribution is also known for design of control commands for pursuer.

iii. $\phi^{E}$ : This is the true normal distribution representing the evader, $E$, and is updated by a Kalman filter using sensor readings of the position of the evader $\left(\left\{z_{0}^{E}, z_{1}^{E}, z_{2}^{E}, \cdots\right\}\right)$ as well as the control commands issued by the pursuer $\left(\left\{d_{0}^{E}, d_{1}^{E}, d_{2}^{E}, \cdots\right\}\right)$. This distribution is not known to the controller in designing the control commands, and is only used in our theoretical analysis to prove that the distribution is within a bounded distance from $\hat{\phi}^{E}$.

We assume that the observation model and the system dynamics model of both the evader and the pursuer are known for designing the control commands for the pursuer.

First, we will show that in spite of partial knowledge of the sequence of inputs, the distance between $\phi^{E}$ and $\hat{\phi}^{E}$ remains bounded under certain assumptions as time goes to infinity. Then we will show that the distance between estimated evader and the pursuer is also bounded. For these we will use two distinct notions of distances between distributions - a metric in the space of normal distributions, and the $\mathcal{F}$-distance, which is a distance function not satisfying the coincidence axiom of a metric.

\section{PRELIMinaries}

\section{A. Normal distributions}

We consider non-degenerate normal distributions on $\mathbb{R}^{n}$ with the standard Euclidean metric. Upon fixing a coordinate on $\mathbb{R}^{n}$, such a distribution, $\phi$, is completely described by a mean, $\mu \in \mathbb{R}^{n}$, and a covariance matrix, $\Sigma \in \operatorname{Sym}^{+}(n, \mathbb{R})$. In particular, we write $\phi=\mathcal{N}(\mu, \Sigma)$, and the explicit formula for the distribution is given by

$\phi(x)=\frac{1}{\sqrt{(2 \pi)^{n}|\Sigma|}} \exp \left[-\frac{1}{2}(x-\mu)^{T} \Sigma^{-1}(x-\mu)\right], \quad x \in \mathbb{R}^{n}$

where, $|\Sigma|$ is the determinant of the covariance matrix.

We restrict attention to the space of non-degenerate normal distributions, which is isomorphic to $\mathbb{R}^{n} \times \operatorname{Sym}^{+}(n, \mathbb{R})$. We denote this space of normal distributions on $\mathbb{R}^{n}$ by $\mathscr{N}^{n} \subset \mathscr{P}\left(\mathbb{R}^{n}\right)$ (where $\mathscr{P}\left(\mathbb{R}^{n}\right)$ is the space of all probability distributions on $\left.\mathbb{R}^{n}\right)$. Thus, $\phi=\mathcal{N}(\mu, \Sigma) \in \mathscr{N}^{n}$.

\section{B. Kalman filters}

Suppose the system dynamics and measurement models [9] are described by

$$
\begin{aligned}
z_{k} & =H x_{k}+v_{k} \\
x_{k+1} & =F x_{k}+B d_{k}+w_{k}
\end{aligned}
$$

where, $x_{k} \in \mathbb{R}^{n}$ is the system state; for $m \leq n, z_{k} \in \mathbb{R}^{m}$ is the measurement (or observations) taken by an observer; $d_{k} \in \mathbb{R}^{q}$ is the input; and $w_{k}$ and $v_{k}$ are the system and measurement noises respectively with the joint covariance matrix $E\left(\left[\begin{array}{c}w_{k} \\ v_{k}\end{array}\right]\left[w_{k}^{T} v_{k}^{T}\right]\right)=\left[\begin{array}{cc}Q & S \\ S^{T} & R\end{array}\right]$. By writing the the above equation in the particular order we emphasize that in designing control command, $d_{k}$, at the $k^{t h}$ time-step, the observation $z_{k}$ is available.

The Kalman filter associated with this system, and its optimality and stability properties are well-known [7]. In particular, if the distribution of $x_{k}$ is the normal distribution with mean $\mu_{k}$ and covariance $\Sigma_{k}$, we define the mean and covariance of the posterior distribution as $m_{k}=F \mu_{k}$ and $V_{k}=F \Sigma_{k} F^{T}+Q$. Then $m_{k}$ and $V_{k}$ evolve according to the following (time-invariant) Kalman filter recursion:

$$
\begin{aligned}
& m_{k+1}=\left(F-\Delta_{k} H\right)\left(m_{k}+A u_{k}+B d_{k}\right)+\Delta_{k} z_{k} \\
& V_{k+1}=\left(F-\Delta_{k} H\right) V_{k}\left(F-\Delta_{k} H\right)^{T} \\
&+\left(Q-S R^{-1} S^{T}\right) \\
&+\left(\Delta_{k}-S R^{-1}\right) R\left(\Delta_{k}-S R^{-1}\right)^{T}
\end{aligned}
$$

$\Delta_{k}$ is often called the Kalman gain and is given by

$$
\Delta_{k}=\left(F V_{k} H^{T}+S\right)\left(H V_{k} H^{T}+R\right)^{-1}
$$

In the literature there are several equivalent formulations of the filter in use, and often the problem is simplified by assuming $S=0$. Our current formulation is closely related to that presented in [7].

\section{Notations}

Note that for a given set of system and measurement parameters, $\{F, H, B, Q, R, S\}$, the sequences $\left\{m_{0}, m_{1}, \cdots\right\}$ and $\left\{V_{0}, V_{1}, \cdots\right\}$ are completely determined by the initial values $\left(m_{0}\right.$ and $\left.V_{0}\right)$ and the sequences $\left\{u_{0}, u_{1}, \cdots\right\}$, $\left\{d_{0}, d_{1}, \cdots\right\}$ and $\left\{z_{0}, z_{1}, \cdots\right\}$. Also, assuming $F$ to be of full rank, $\mu_{k}=F^{-1} m_{k}$ and $\Sigma_{k}=F^{-1}\left(V_{k}-Q\right) F^{-T}$ gives the prior distribution.

We will use the following notations for brevity:

i. $\left\{\phi_{k}\right\}_{k=0,1,2, \ldots}$ be the sequence of normal distributions with mean $\mu_{k}$ and covariance $\Sigma_{k}$ which evolve according to the equations (3) and (4).

ii. The set of parameters governing the evolution of the distribution, $\mathcal{P}=\{F, H, B, Q, R, S\}$.

iii. The Kalman filter can be thought of a function that takes in the parameter set, the initial conditions, the sequence of control commands and the sequence of observations, and generates the sequence of distributions, and hence can be compactly written as either of the following:

$$
\begin{aligned}
& \left\{m_{k}, V_{k}\right\}_{k=0,1,2, \ldots}= \\
& \mathcal{K \mathcal { F }}_{\mathcal{P}}^{\text {post }}\left(\left\{m_{0}, V_{0}\right\} ;\left\{d_{k}\right\}_{k=0,1,2, \cdots} ;\left\{z_{k}\right\}_{k=0,1,2, \cdots}\right) \\
& \left\{\phi_{k}\right\}_{k=0,1,2, \ldots}= \\
& \mathcal{K \mathcal { F }}_{\mathcal{P}}^{\text {pri }}\left(\left\{m_{0}, V_{0}\right\} ;\left\{d_{k}\right\}_{k=0,1,2, \cdots} ;\left\{z_{k}\right\}_{k=0,1,2, \cdots) .}\right.
\end{aligned}
$$

\section{Riccati equation}

It can be observed that the evolution of the covariance matrix according to equation (4) depends only on the system parameters and the initial covariance matrix $V_{0}$. It does not depend on $m_{0}$, the inputs $\left\{u_{k}, d_{k}\right\}_{k=0,1,2, \ldots}$, or the

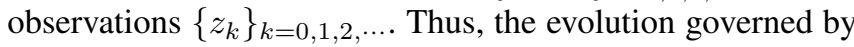
equation (4) is often studied separately and is called the discrete-time Matrix Riccati equation. Results related to its convergence, stability and uniqueness of the limiting value are well-known and summarized next. 
Definition 1 (Stabilizable pair): For matrices $F$ and $G$, $(F, G)$ is called a stabilizable pair if there exists a matrix $K$ such that all the eigenvalues of $F-G K$ have absolute values less than unity.

Definition 2 (Detectable pair): For matrices $H$ and $F$, $(H, F)$ is called a detectable pair if there exists a matrix $K$ such that all the eigenvalues of $F-K H$ have absolute values less than unity.

Note: stabilizability and detectability are generic conditions - almost all pairs (except a set of measure zero) are stabilizable as well as detectable.

\section{Lemma 1 (Matrix Riccati equation [7], [1]):}

a. (Convergence) If $(H, F)$ is a detectable pair, then the sequence $\left\{V_{k}\right\}_{k=0,1,2, \ldots}$ (generated by Eqn. (4)) converges to $V_{\infty}-$ a finite matrix that satisfies the algebraic Riccati equation:

$$
\begin{aligned}
& V=\tilde{F}(V) V \tilde{F}(V)^{T}+\left(Q-S R^{-1} S^{T}\right) \\
& \quad+\left(\Delta(V)-S R^{-1}\right) R\left(\Delta(V)-S R^{-1}\right)^{T}
\end{aligned}
$$

where, $\tilde{F}(V)=F-\left(F V H^{T}+S\right)\left(H V H^{T}+R\right)^{-1} H$, and

$$
\Delta(V)=\left(F V H^{T}+S\right)\left(H V H^{T}+R\right)^{-1}
$$

b. (Stability and Uniqueness) If $(F, G)$ is a stabilizable pair for any $G$ such that $G G^{T}=Q$, then the solution $V_{\infty}$ of (6) to which $\left\{V_{k}\right\}_{k=0,1,2, \cdots}$ converges to is unique (i.e., independent of the initial choice of $V_{0}$ ) and $F-\Delta\left(V_{\infty}\right) H$ is stable, that is, the spectral radius $\rho\left(F-\Delta\left(V_{\infty}\right) H\right)<1$.

\section{E. Metrics and Distance Functions on Space of Normal Distributions}

1) Product Metric: Consider the problem of measuring the distance between the true distribution of the evader, $\phi_{k}^{E}$, and its estimated distribution, $\hat{\phi}_{k}^{E}$. If the distributions are exactly the same we could say that the estimate is perfect, and this fact should be reflected by our equipment for measuring their distances. Thus it is natural to use a metric, $\mathcal{D}: \mathscr{N}^{n} \times \mathscr{N}^{n} \rightarrow \mathbb{R}_{+}$, so that $\mathcal{D}\left(\phi^{E}, \hat{\phi}^{E}\right)=0$ if and only if $\phi^{E}=\hat{\phi}^{E}$ and satisfies the other axioms of metric. The simple choice we make is the product metric of the Euclidean metric on the space of means of the normal distributions and the induced 2-norm of the difference of matrices as the metric on the space of the normal distributions' covariance matrices. Thus,

$$
\mathcal{D}\left(\phi_{k}^{E}, \hat{\phi}_{k}^{E}\right)=\sqrt{\left\|F\left(\mu_{k}-\hat{\mu}_{k}\right)\right\|^{2}+\left\|F^{T}\left(\Sigma_{k}-\hat{\Sigma}_{k}\right) F\right\|^{2}}
$$

where, $\phi_{k}^{E}=\mathcal{N}\left(\mu_{k}^{E}, \Sigma_{k}^{E}\right)$ and $\hat{\phi}_{k}^{E}=\mathcal{N}\left(\hat{\mu}_{k}^{E}, \hat{\Sigma}_{k}^{E}\right)$

2) $\mathcal{F}$-distance: Next consider the problem of measuring the distance between the pursuer and the estimated evader. By "distance" we would like to measure an analogue of the expected distance between the position of the pursuer and that of the evader. The interesting point to note here is that a metric will not suffice, since when the distributions $\phi_{k}^{P}$ and $\hat{\phi}_{k}^{E}$ are exactly same, the expected distances won't be zero. Thus we define the $\mathcal{F}$-distance, $\mathcal{F}: \mathscr{P}\left(\mathbb{R}^{n}\right) \times \mathscr{P}\left(\mathbb{R}^{n}\right) \rightarrow \mathbb{R}_{+}$,

$$
\mathcal{F}\left(\phi_{k}^{P}, \hat{\phi}_{k}^{E}\right)=\sqrt{\int_{\mathbb{R}^{n}} \int_{\mathbb{R}^{n}} \phi_{k}^{P}(x) \hat{\phi}_{k}^{E}(y)(d(x, y))^{2} \mathrm{~d} x \mathrm{~d} y}
$$

This, in essence, is similar to the more wellknown Lukaszyk-Karmowski distance [6], $\operatorname{LK}\left(\phi_{1}, \phi_{2}\right)=$
$\int_{\mathbb{R}^{n}} \int_{\mathbb{R}^{n}} \phi_{1}(x) \phi_{2}(y) \quad d(x, y) \mathrm{d} x \mathrm{~d} y$, but computationally more favorable. In particular, $\mathcal{F}$ has the following properties (see Appendix for proofs):

i. For $\phi_{k}^{P}=\mathcal{N}\left(\mu_{k}^{P}, \Sigma_{k}^{P}\right)$ and $\hat{\phi}_{k}^{E}=\mathcal{N}\left(\hat{\mu}_{k}^{E}, \hat{\Sigma}_{k}^{E}\right)$, $\mathcal{F}\left(\phi_{k}^{P}, \hat{\phi}_{k}^{E}\right)=\sqrt{\operatorname{Tr}\left(\Sigma_{k}^{P}\right)+\operatorname{Tr}\left(\hat{\Sigma}_{k}^{E}\right)+\left\|\mu_{k}^{P}-\hat{\mu}_{k}^{E}\right\|^{2}}$, and,

ii. $\mathcal{F}$ satisfies triangle inequality: $\mathcal{F}\left(\phi_{1}, \phi_{3}\right) \leq$ $\mathcal{F}\left(\phi_{1}, \phi_{2}\right)+\mathcal{F}\left(\phi_{2}, \phi_{3}\right), \forall \phi_{i} \in \mathscr{N}^{n}$

\section{Main Results}

Our principal results are split into two parts: $i$. Proving that $\lim _{k \rightarrow \infty} \mathcal{D}\left(\phi_{k}^{E}, \hat{\phi}_{k}^{E}\right)$ is bounded (Proposition 1), and, ii. designing the control commands, $d_{k}^{P}, k=0,1, \cdots$, which dictate the evolution of $\phi_{k}^{P}$ with $k$, and hence prove that $\lim _{k \rightarrow \infty} \mathcal{F}\left(\phi_{k}^{P}, \hat{\phi}_{k}^{E}\right)$ is bounded (Proposition 2). A final corollary concludes the paper by establishing that $\lim _{k \rightarrow \infty} \mathcal{F}\left(\phi_{k}^{P}, \phi_{k}^{E}\right)$ is bounded as well.

For clarity, we present abridged statements of the main propositions in this section, while the detailed statements and proofs are presented in the Appendix.

\section{A. Distance between real/true evader and estimated evader}

For a symmetric matrix $Q, Q \geqslant 0$ means that $Q$ is a positive semi-definite.

Lemma 2: [7] Let $(H, F)$ be a detectable pair (Definition 2). Let $\left\{A_{k}\right\}$ be a sequence generated by:

$$
\begin{aligned}
A_{k+1}= & (F-K H) A_{k}(F-K H)^{T}+\left(Q-S R^{-1} S^{T}\right) \\
& +\left(K-S R^{-1}\right) R\left(K-S R^{-1}\right)^{T} .
\end{aligned}
$$

If $A_{0}=V_{0}$, then $V_{k} \leqslant A_{k}$ (note: $\left\{V_{k}\right\}$ is generated by (4)).

Proposition 1: Suppose $\mathcal{P}=\{F, H, B, Q, R, S\}$ is the set of model parameters that govern the evolution of the distributions $\phi_{k}^{E}$ and $\hat{\phi}_{k}^{E}$. Consider the following Kalman Filter sequences for the evolution of the evader's true and estimated distributions:

$$
\begin{aligned}
& \left.\left\{\phi_{k}^{E}\right\}_{k=0,1,2, \cdots}=\mathcal{K F}_{\mathcal{P}}^{p r i}\left(\left\{m_{0}, V_{0}\right\} ;\left\{d_{k}\right\}_{k=0,1,2, \ldots ;} ; z_{k}\right\}_{k=0,1,2, \ldots}\right)
\end{aligned}
$$

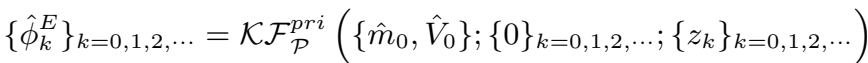

If $(F, H)$ is detectable, and $(F, G)$ is a stabilizable pair for any $G$ such that $G G^{T}=Q$, and if $\left\|B d_{k}\right\| \leq c_{1}, \| H m_{k}-$ $z_{k} \| \leq c_{2}$ and $\left\|F-\Delta\left(V_{\infty}\right) H\right\|<1$ for some positive constants $c_{1}, c_{2}$, then $\lim _{k \rightarrow \infty} \mathcal{D}\left(\phi_{k}^{E}, \hat{\phi}_{k}^{E}\right)$ is bounded.

The assumption $\left\|B d_{k}\right\| \leq c_{1}$ simply implies that the evader cannot move arbitrarily fast. The statement $\| H m_{k}-$ $z_{k} \| \leq c_{2}$ is justified by the fact that $z_{k}$, the sensor measurement of the true position of the evader, is a sample from a distribution whose estimated mean is $F \mu_{k}=m_{k}$. The $H$ appears due to the transformation of the position coordinates into the sensor's measurement coordinates. The final condition is a modestly stronger requirement than $(F, G)$ be stable.

As consequence of the above proposition, we can guarantee that our estimate $\hat{\phi}_{k}^{E}$ of the evader's distribution, computed without the knowledge of the control commands issued by the evader, will remain within a finite distance of the true distribution even as $k \rightarrow \infty$. 


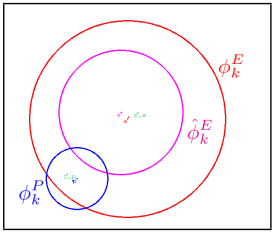

(a) $k=1$

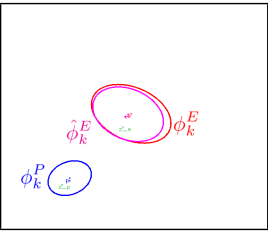

(b) $k=3$

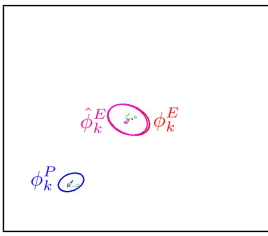

(c) $k=11$

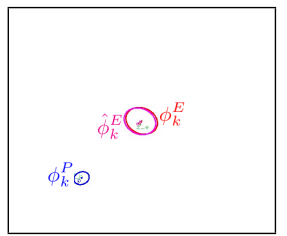

(d) $k=50$

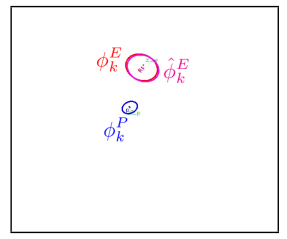

(e) $k=501$

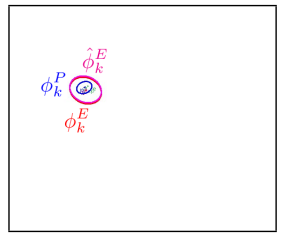

(f) $k=1001$

Fig. 2. Snapshots of simulation at different values of $k$. The three ellipses represent the covariance and mean of the distributions $\hat{\phi}_{k}^{E}, \phi_{k}^{E}$ and $\phi_{k}^{P}$.

\section{B. Pursuit-Evasion on distributions}

Proposition 2: Let $\phi_{k}^{P}$ be the normal distribution describing the pursuer, which evolves according to the Kalman filter

$$
\left.\mathcal{K F}_{\mathcal{P} P}^{p r i}\left(\left\{m_{0}^{P}, V_{0}^{P}\right\} ;\left\{d_{k}^{P}\right\}_{k=0,1,2, \cdots ;} ; z_{k}^{P}\right\}_{k=0,1,2, \cdots}\right)
$$

where the given set of system and measurement parameters are, $\mathcal{P}^{P}=\left\{F^{P}, H^{P}, A^{P}, B^{P}, Q^{P}, R^{P}, S^{P}\right\}$. Suppose $(F, H)$ is detectable, $(F, G)$ is a stabilizable pair for any $G$ such that $G G^{T}=Q,\left(F^{P}, H^{P}\right)$ detectable and $\left(F^{P}, G^{P}\right)$ is a stabilizable pair for any $G^{P}$ such that $G^{P}\left(G^{P}\right)^{T}=Q^{P}$, $\left\|B d_{k}\right\| \leq c_{1},\left\|H m_{k}-z_{k}\right\| \leq c_{2},\left\|F-\Delta\left(V_{\infty}\right) H\right\|<1$, $\left\|H m_{k}^{P}=z_{k}^{P}\right\| \leq c_{3}$, and $F^{P}-\Delta\left(V_{\infty}^{P}\right) H^{P}$ is invertible. If the pursuer issues the control command

$$
\begin{aligned}
d_{k}^{P}=\left(B^{P}\right)^{-1}\left(F^{P}-\Delta_{k}^{P} H^{P}\right)^{-1}\left[\left(F-\hat{\Delta}_{k} H\right) m_{k}^{P}\right. \\
\left.-\left(F^{P}-\Delta_{k}^{P} H^{P}\right) m_{k}^{P}+\hat{\Delta}_{k} H \hat{m}_{k}-\Delta_{k}^{P} H m_{k}^{P}\right],
\end{aligned}
$$

then $\lim _{k \rightarrow \infty} \mathcal{F}\left(\phi_{k}^{P}, \hat{\phi}_{k}^{E}\right)$ is bounded.

The conditions $\left\|B d_{k}\right\| \leq c_{1},\left\|H m_{k}-z_{k}\right\| \leq c_{2}$ and $\| F-$ $\Delta\left(V_{\infty}\right) H \|<1$ are same as Proposition 1. $\left\|H m_{k}^{P}-z_{k}^{P}\right\| \leq$ $c_{3}$ is justified due to the fact that $z_{k}^{P}$ is an observation made from the pursuer's distribution. The invertibility of $F^{P}-$ $\Delta\left(V_{\infty}^{P}\right) H^{P}$ is once again a weak assumption.

Due to the way we define of the subscripts of measurements, (2), we note that this design of $d_{k}$ respects causality.

\section{Bound on the pursuer-evader distance}

Corollary 1: If the distributions of the true evader $\phi_{k}^{E}$, the estimated evader $\hat{\phi}_{k}^{E}$ and the pursuer $\phi_{k}^{P}$ evolve according to the conditions given in Proposition 1 and 2, then $\lim _{k \rightarrow \infty} \mathcal{F}\left(\phi_{k}^{P}, \phi_{k}^{E}\right)$ is bounded.

This concludes our result on the claimed boundedness.

\section{Simulation}

We implemented the Kalman filter and the described control in $\mathrm{C}++$ programming language. In this section we present the result of the simulations.

For the simulation result presented in this paper, we choose the following parameters for the dynamics and measurement models of the evader, and the initial conditions for the evader's true distribution:

$\mu_{0}=\left[\begin{array}{l}0 \\ 0\end{array}\right], \quad \Sigma_{0}=\left[\begin{array}{ll}5 & 0 \\ 0 & 5\end{array}\right], F=\left[\begin{array}{ll}1 & 0 \\ 0 & 1\end{array}\right], B=\left[\begin{array}{cc}0.01 & 0 \\ 0 & 0.01\end{array}\right]$,

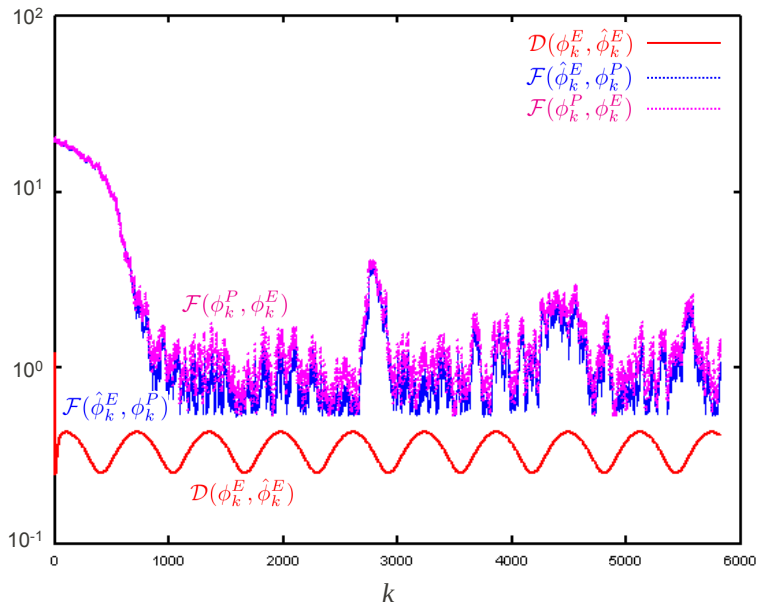

Fig. 3. The distances $\mathcal{D}\left(\phi_{k}^{E}, \hat{\phi}_{k}^{E}\right), \mathcal{F}\left(\hat{\phi}_{k}^{E}, \phi_{k}^{P}\right)$ and $\mathcal{F}\left(\phi_{k}^{P}, \phi_{k}^{E}\right)$ plotted against $k$. (note that the $Y$ axis has a logarithmic scale).

$H=\left[\begin{array}{ll}1 & 0 \\ 0 & 1\end{array}\right], Q=\left[\begin{array}{cc}0.01 & 0 \\ 0 & 0.01\end{array}\right], R=\left[\begin{array}{ll}2.0 & 0.5 \\ 0.5 & 1.0\end{array}\right], S=\left[\begin{array}{ll}0 & 0 \\ 0 & 0\end{array}\right]$

For the evader estimate we choose,

$\hat{\mu}_{0}^{E}=\left[\begin{array}{c}0.1 \\ -0.1\end{array}\right], \quad \hat{\Sigma}_{0}^{E}=\left[\begin{array}{ll}1 & 0 \\ 0 & 1\end{array}\right]$,

$\hat{F}=F, \hat{B}=B, \hat{H}=H, \hat{Q}=Q, \hat{R}=R, \hat{S}=S$

and for the pursuer,

$\mu_{0}^{P}=\left[\begin{array}{l}-10 \\ -15\end{array}\right], \quad \Sigma_{0}^{P}=\left[\begin{array}{ll}5 & 0 \\ 0 & 5\end{array}\right], F^{P}=\left[\begin{array}{ll}1 & 0 \\ 0 & 1\end{array}\right]$,

$B^{P}=\left[\begin{array}{cc}0.01 & 0 \\ 0 & 0.01\end{array}\right], H^{P}=\left[\begin{array}{ll}1 & 0 \\ 0 & 1\end{array}\right]$,

$Q^{P}=\left[\begin{array}{cc}0.001 & 0 \\ 0 & 0.001\end{array}\right], R^{P}=\left[\begin{array}{cc}1.0 & -0.25 \\ -0.25 & 0.5\end{array}\right], S^{P}=\left[\begin{array}{ll}0 & 0 \\ 0 & 0\end{array}\right]$

With these parameters the snapshots of the simulation results obtained is shown in Figure 2, while the plot in Figure 3 shows how $\mathcal{D}\left(\phi_{k}^{E}, \hat{\phi}_{k}^{E}\right), \mathcal{F}\left(\phi_{k}^{P}, \hat{\phi}_{k}^{E}\right)$ and $\mathcal{D}\left(\hat{\phi}_{k}^{E}, \phi_{k}^{P}\right)$ varies with $k$. Although there is almost random oscillation in the values of $\mathcal{F}\left(\phi_{k}^{P}, \hat{\phi}_{k}^{E}\right)$ and $\mathcal{D}\left(\hat{\phi}_{k}^{E}, \phi_{k}^{P}\right)$, the bounds are clearly noticeable even for large values of $k$. 


\section{APPENDIX}

\section{A. Proof of claimed properties of $\mathcal{F}$}

Statement: $\mathcal{F}\left(\phi_{1}, \phi_{2}\right)=\sqrt{\operatorname{Tr}\left(\Sigma_{1}\right)+\operatorname{Tr}\left(\Sigma_{2}\right)+\left\|\mu_{1}-\mu_{2}\right\|^{2}}$ and $\mathcal{F}\left(\phi_{1}, \phi_{3}\right) \leq \mathcal{F}\left(\phi_{1}, \phi_{2}\right)+\mathcal{F}\left(\phi_{2}, \phi_{3}\right)$ for all $\phi_{i}=\mathcal{N}\left(\mu_{i}, \Sigma_{i}\right) \in$ $\mathscr{N}^{N}$.

Proof: From the definitions, $\mathcal{F}\left(\phi_{1}, \phi_{2}\right)=\sqrt{\int_{\mathbb{R}^{n}} \int_{\mathbb{R}^{n}} \phi_{1}\left(x_{1}\right) \phi_{2}\left(x_{2}\right)\left\|x_{1}-x_{2}\right\|^{2} \mathrm{~d} x_{1} \mathrm{~d} x_{2}}$, $x_{i} \in \mathbb{R}^{N}$. By plugging in $\left\|x_{1}-x_{2}\right\|^{2}=\sum_{i=1,2}\left\|x_{i}-\mu_{i}\right\|^{2}+$ $\left\|\mu_{1}-\mu_{2}\right\|^{2}-2\left(x_{1}-\mu_{1}\right)^{T}\left(x_{2}-\mu_{2}\right)+2\left(\mu_{1}-\mu_{2}\right)^{T}\left(\left(x_{1}-\mu_{1}\right)-\right.$ $\left.\left(x_{2}-\mu_{2}\right)\right)$ into the above, and noting that: (1) $\int_{\mathbb{R}^{n}} \phi_{i}\left(x_{i}\right) \mathrm{d} x_{i}=1$; (2) $\int_{\mathbb{R}^{n}} \phi_{i}\left(x_{i}\right)\left\|x_{i}-\mu_{i}\right\|^{2} \mathrm{~d} x_{i}=\operatorname{Tr}\left(\Sigma_{i}\right)$; and (3) $\int_{\mathbb{R}^{n}} \phi_{i}\left(x_{i}\right)\left(x_{i}-\right.$ $\left.\mu_{i}\right) \mathrm{d} x_{i}=\mathbf{0}$, the proof for the first part follows immediately.

Using this result we have

$$
\left(\mathcal{F}\left(\phi_{1}, \phi_{2}\right)+\mathcal{F}\left(\phi_{2}, \phi_{3}\right)\right)^{2}=
$$

$$
=\operatorname{Tr}\left(\Sigma_{1}\right)+2 \operatorname{Tr}\left(\Sigma_{2}\right)+\operatorname{Tr}\left(\Sigma_{3}\right)+\left\|\mu_{1}-\mu_{2}\right\|^{2}+\left\|\mu_{2}-\mu_{3}\right\|^{2}
$$

$$
+2 \sqrt{\left(\begin{array}{rr}
\left.\operatorname{Tr}\left(\Sigma_{1}\right)+\operatorname{Tr}\left(\Sigma_{2}\right)+\left\|\mu_{1}-\mu_{2}\right\|^{2}\right) \\
\left(\operatorname{Tr}\left(\Sigma_{2}\right)+\operatorname{Tr}\left(\Sigma_{3}\right)+\left\|\mu_{2}-\mu_{3}\right\|^{2}\right)
\end{array}\right.}
$$

$\geq \operatorname{Tr}\left(\Sigma_{1}\right)+\operatorname{Tr}\left(\Sigma_{3}\right)+\left(\left\|\mu_{1}-\mu_{2}\right\|+\left\|\mu_{2}-\mu_{3}\right\|\right)^{2}$

$$
\geq \operatorname{Tr}\left(\Sigma_{1}\right)+\operatorname{Tr}\left(\Sigma_{3}\right)+\left\|\mu_{1}-\mu_{3}\right\|^{2}=\left(\mathcal{F}\left(\phi_{1}, \phi_{3}\right)\right)^{2}
$$

\section{B. Proof of Proposition 1}

Statement of Proposition 1: Suppose $\mathcal{P}=\{F, H, B, Q, R, S\}$ is the set of model parameters that govern the evolution of the distributions $\phi_{k}^{E}$ and $\hat{\phi}_{k}^{E}$. Consider the following Kalman Filter sequences for the evolution of the evader's true and estimated distributions:

$$
\begin{aligned}
& \left\{\phi_{k}^{E}\right\}_{k=0,1,2, \ldots}=\mathcal{K} \mathcal{F}_{\mathcal{P}}^{\text {pri }}\left(\left\{m_{0}, V_{0}\right\} ;\left\{d_{k}\right\}_{k=0,1,2, \ldots} ;\left\{z_{k}\right\}_{k=0,1,2, \ldots)}\right.
\end{aligned}
$$

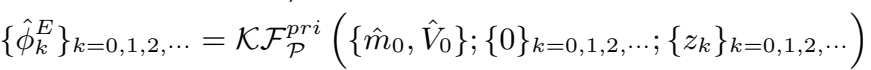

If $(F, H)$ is detectable, and $(F, G)$ is a stabilizable pair for any $G$ such that $G G^{T}=Q$, and if $\left\|B d_{k}\right\| \leq c_{1},\left\|H m_{k}-z_{k}\right\| \leq c_{2}$ and $\left\|F-\Delta\left(V_{\infty}\right) H\right\|<1$ for some positive constants $c_{1}, c_{2}$, then there is a finite constant $C\left(V_{0}, \hat{V}_{0}\right)$ such that $\lim _{k \rightarrow \infty} \mathcal{D}\left(\phi_{k}^{E}, \hat{\phi}_{k}^{E}\right) \leq$ $\left\|m_{0}-\hat{m}_{0}\right\|+C\left(V_{0}, \hat{V}_{0}\right)$.

Proof: First we write down the expression for $\mathcal{D}\left(\phi_{k}^{E}, \hat{\phi}_{k}^{E}\right)$ :

$$
\mathcal{D}\left(\phi_{k}^{E}, \hat{\phi}_{k}^{E}\right)=\sqrt{\left\|m_{k}-\hat{m}_{k}\right\|^{2}+\left\|F^{T}\left(\Sigma_{k}-\hat{\Sigma}_{k}\right) F\right\|^{2}} \text {. }
$$

By definition, $V_{k}=F \Sigma_{k} F^{T}+Q$ and $\hat{V}_{k}=F \hat{\Sigma}_{k} F^{T}+Q$. By Lemma $1, \lim _{k \rightarrow \infty} V_{k}=\lim _{k \rightarrow \infty} \hat{V}_{k}=V_{\infty}$. Thus the second term under the square root in (12) vanishes as $k \rightarrow \infty$.

Next, $\hat{\Delta}_{k}$, the Kalman gain of an estimated evader is given by

$$
\hat{\Delta}_{k}=\left(F \hat{V}_{k} H^{T}+S\right)\left(H \hat{V}_{k} H^{T}+R\right)^{-1} \text {. }
$$

Since $m_{k}=\left(F-\Delta_{k-1} H\right)\left(m_{k-1}+B d_{k-1}\right)+\Delta_{k-1} z_{k-1}$ and $\hat{m}_{k}=\left(F-\hat{\Delta}_{k-1} H\right) \hat{m}_{k-1}+\hat{\Delta}_{k-1} z_{k-1}$ by definition,

$$
m_{k}-\hat{m}_{k}=\left(F-\hat{\Delta}_{k-1} H\right)\left(m_{k-1}-\hat{m}_{k-1}\right)+
$$

$$
\left(F-\Delta_{k-1} H\right) B d_{k-1}+\left(\hat{\Delta}_{k-1}-\Delta_{k-1}\right)\left(H m_{k-1}-z_{k-1}\right)
$$

\section{Inducting on this yields:}

$$
\begin{aligned}
& m_{k}-\hat{m}_{k}=\prod_{i=0}^{k-1}\left(F-\hat{\Delta}_{k-1-i} H\right)\left(m_{0}-\hat{m}_{0}\right) \\
& \quad+\sum_{i=0}^{k-1}\left[\prod_{j=0}^{i-1}\left(F-\hat{\Delta}_{k-1-j} H\right)\right]\left(F-\Delta_{k-1-i} H\right) B d_{k-1-i} \\
& +\sum_{i=0}^{k-1}\left[\prod_{j=0}^{i-1}\left(F-\hat{\Delta}_{k-1-j} H\right)\right]\left(\hat{\Delta}_{k-1-i}-\Delta_{k-1-i}\right) e_{k-1-i}
\end{aligned}
$$

where $e_{k}=H m_{k}-z_{k}$ and $\prod_{j=0}^{-1}=I d$. Since $\lim _{k \rightarrow \infty} \|(F-$ $\left.\Delta\left(V_{\infty}\right) H\right)^{k} \|^{1 / k}=\rho\left(F-\Delta\left(V_{\infty}\right) H\right)<1$ by Lemma 1 , we have

$$
\begin{aligned}
\lim _{k \rightarrow \infty}\left\|m_{k}-\hat{m}_{k}\right\| \leq\left\|m_{0}-\hat{m}_{0}\right\| \\
\quad+c_{1} \lim _{k \rightarrow \infty} \sum_{i=0}^{k-1}\left\|\left[\prod_{j=0}^{i-1}\left(F-\hat{\Delta}_{k-1-j} H\right)\right]\left(F-\Delta_{k-1-i} H\right)\right\| \\
\quad+c_{2} \lim _{k \rightarrow \infty} \sum_{i=0}^{k-1}\left\|\left[\prod_{j=0}^{i-1}\left(F-\hat{\Delta}_{k-1-j} H\right)\right]\left(\hat{\Delta}_{k-1-i}-\Delta_{k-1-i}\right)\right\| .
\end{aligned}
$$

We need to show that two limits in the previous inequality are bounded. Since $\left\|F-\Delta\left(V_{\infty}\right) H\right\|<1$, there exists a constant $\epsilon>0$ such that $\left\|F-\Delta\left(V_{\infty}\right) H\right\|+\epsilon<1$. Since $\lim _{k \rightarrow \infty} \Delta_{k}=\Delta\left(V_{\infty}\right)$ and $\lim _{k \rightarrow \infty} \hat{\Delta}_{k}=\Delta\left(V_{\infty}\right)$, there exists a positive integer $k_{0}=$ $k_{0}(\epsilon)$ such that $\left\|F-\Delta_{k} H\right\|-\left\|F-\Delta\left(V_{\infty}\right) H\right\|<\epsilon$ and $\| F-$ $\hat{\Delta}_{k} H\|-\| F-\Delta\left(V_{\infty}\right) H \|<\epsilon$ for all $k \geq k_{0}$.

2 Consider the terms in the sum $\sum_{i=0}^{k-1} \|\left[\prod_{j=0}^{i-1}(F-\right.$ $\left.\left.\hat{\Delta}_{k-1-j} H\right)\right]\left(F-\Delta_{k-1-i} H\right) \|$ when $k \geq k_{0}$. The first term is $\left\|F-\Delta_{k-1} H\right\|$ (since $i=0$ ), and is bounded by $\left\|F-\Delta\left(V_{\infty}\right) H\right\|+\epsilon$. The second term is $\left\|\left(F-\hat{\Delta}_{k-1} H\right)\left(F-\Delta_{k-2} H\right)\right\|$ (since $i=1$ ), and is bounded by $\left(\left\|F-\Delta\left(V_{\infty}\right) H\right\|+\epsilon\right)^{2}$. Following similar argument, sum from $i=0$ to $i=k-k_{0}-1$ is bounded by $\sum_{i=0}^{k-k_{0}-1}\left(\left\|F-\Delta\left(V_{\infty}\right) H\right\|+\epsilon\right)^{i+1}$. Since $\sum_{i=0}^{\infty}\left(\left\|F-\Delta\left(V_{\infty}\right) H\right\|+\epsilon\right)^{i+1}$ is a constant, the limit $\lim _{k \rightarrow \infty} \sum_{i=0}^{k-1}\left\|\left[\prod_{j=0}^{i-1}\left(F-\hat{\Delta}_{k-1-j} H\right)\right]\left(F-\Delta_{k-1-i} H\right)\right\|$ exists and is bounded.

Similarly, since $\Delta\left(V_{\infty}\right)=\lim _{k \rightarrow \infty} \hat{\Delta}_{k}=\lim _{k \rightarrow \infty} \Delta_{k}$, the limit $\lim _{k \rightarrow \infty} \sum_{i=0}^{k-1}\left\|\left[\prod_{j=0}^{i-1}\left(F-\hat{\Delta}_{k-1-j} H\right)\right]\left(\hat{\Delta}_{k-1-i}-\Delta_{k-1-i}\right)\right\|$ exists and is bounded. Thus we have the proposed constant

$$
\begin{array}{r}
C\left(V_{0}, \hat{V}_{0}\right)=c_{1} \lim _{k \rightarrow \infty} \sum_{i=0}^{k-1}\left\|\left[\prod_{j=0}^{i-1}\left(F-\hat{\Delta}_{k-1-j} H\right)\right]\left(F-\Delta_{k-1-i} H\right)\right\| \\
\quad+c_{2} \lim _{k \rightarrow \infty} \sum_{i=0}^{k-1}\left\|\left[\prod_{j=0}^{i-1}\left(F-\hat{\Delta}_{k-1-j} H\right)\right]\left(\hat{\Delta}_{k-1-i}-\Delta_{k-1-i}\right)\right\| .
\end{array}
$$

\section{Proof of Proposition 2}

Statement of Proposition 2: Let $\phi_{k}^{P}$ be the normal distribution describing the pursuer, which evolves according to the Kalman filter $\left\{\phi_{k}^{P}\right\}_{k=0,1, \cdots}=\mathcal{K}_{\mathcal{F}^{P}}^{\text {pri }}\left(\left\{m_{0}^{P}, V_{0}^{P}\right\} ;\left\{d_{k}^{P}\right\}_{k=0,1, \cdots} ;\left\{z_{k}^{P}\right\}_{k=0,1, \cdots}\right)$ where the given set of system and measurement parameters are, $\mathcal{P}^{P}=\left\{F^{P}, H^{P}, A^{P}, B^{P}, Q^{P}, R^{P}, S^{P}\right\}$. Suppose $(F, H)$ is detectable, $(F, G)$ is a stabilizable pair for any $G$ such that $G G^{T}=Q$, $\left(F^{P}, H^{P}\right)$ detectable and $\left(F^{P}, G^{P}\right)$ is a stabilizable pair for any $G^{P}$ such that $G^{P}\left(G^{P}\right)^{T}=Q^{P},\left\|B d_{k}\right\| \leq c_{1},\left\|H m_{k}-z_{k}\right\| \leq c_{2}$, $\left\|F-\Delta\left(V_{\infty}\right) H\right\|<1,\left\|H m_{k}^{P}-z_{k}^{P}\right\| \leq c_{3}$, and $F^{P}-\Delta\left(V_{\infty}^{P}\right) H^{P}$ is invertible. If the pursuer issues the control command/input

$$
\begin{aligned}
d_{k}^{P}=( & \left.B^{P}\right)^{-1}\left(F^{P}-\Delta_{k}^{P} H^{P}\right)^{-1}\left[\left(F-\hat{\Delta}_{k} H\right) m_{k}^{P}\right. \\
& \left.-\left(F^{P}-\Delta_{k}^{P} H^{P}\right) m_{k}^{P}+\hat{\Delta}_{k} H \hat{m}_{k}-\Delta_{k}^{P} H m_{k}^{P}\right],
\end{aligned}
$$

then there is an integer $k_{1} \geq 0$ such that

$$
\begin{aligned}
\lim _{k \rightarrow \infty} \mathcal{F}\left(\phi_{k}^{P}, \hat{\phi}_{k}^{E}\right)^{2} \leq & \operatorname{Tr}\left(V_{\infty}\right)+\operatorname{Tr}\left(V_{\infty}^{P}\right) \\
& +\left[\left\|\hat{m}_{k_{1}}-m_{k_{1}}^{P}\right\|+C\left(V_{0}, \hat{V}_{0}, V_{0}^{P}\right)\right]^{2}
\end{aligned}
$$

where the quantity on the right is finite, with $V_{\infty}^{P}=\lim _{k \rightarrow \infty} V_{k}^{P}$ and 


$$
\begin{aligned}
& C\left(V_{0}, \hat{V}_{0}, V_{0}^{P}\right) \\
& =c_{3} \lim _{k \rightarrow \infty} \sum_{i=0}^{k-k_{1}-1}\left\|\left[\prod_{j=0}^{i-1}\left(F-\hat{\Delta}_{k-1-j} H\right)\right] \Delta_{k-1-i}^{P}\right\| \\
& \quad+\lim _{k \rightarrow \infty} \sum_{i=0}^{k-k_{1}-1}\left\|\left[\prod_{j=0}^{i-1}\left(F-\hat{\Delta}_{k-1-j} H\right)\right] \hat{\Delta}_{k-1-i}\left(H \hat{m}_{k-1-i}-z_{k-1-i}\right)\right\|
\end{aligned}
$$

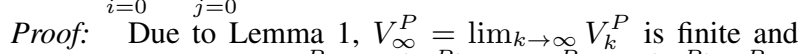
is unique. Since $\lim _{k \rightarrow \infty} \Delta_{k}^{P}=\Delta\left(V_{\infty}^{P}\right)$ and $F^{P}-\Delta\left(V_{\infty}^{P}\right) H^{P}$ is invertible, there is an integer $k_{1} \geq 0$ such that $F^{P}-\Delta_{k}^{P} H^{P}$ is invertible for all $k \geq k_{1}$. Since $m_{k}^{\bar{P}}=\left(F^{P}-\Delta_{k-1}^{P} H^{P}\right)\left(m_{k-1}^{P}+\right.$ $\left.B^{P} d_{k-1}^{P}\right)+\Delta_{k-1}^{P} \bar{z}_{k-1}^{P}$, then for all $k \geq k_{1}$ we have,

$$
\begin{aligned}
\hat{m}_{k}-m_{k}^{P} & =\left(F-\hat{\Delta}_{k-1} H\right)\left(\hat{m}_{k-1}-m_{k-1}^{P}\right) \\
& +\Delta_{k-1}^{P}\left(H m_{k-1}^{P}-z_{k-1}^{P}\right)-\hat{\Delta}_{k-1}\left(H \hat{m}_{k-1}-z_{k-1}\right) .
\end{aligned}
$$

Inducting on this yields:

$$
\begin{aligned}
& \hat{m}_{k}-m_{k}^{P}=\prod_{i=0}^{k-k_{1}-1}\left(F-\hat{\Delta}_{k-1-i} H\right)\left(\hat{m}_{k_{1}}-m_{k_{1}}^{P}\right) \\
& +\sum_{i=0}^{k-k_{1}-1}\left[\prod_{j=0}^{i-1}\left(F-\hat{\Delta}_{k-1-j} H\right)\right] \Delta_{k-1-i}^{P}\left(H m_{k-1-i}^{P}-z_{k-1-i}^{P}\right) \\
& -\sum_{i=0}^{k-k_{1}-1}\left[\prod_{j=0}^{i-1}\left(F-\hat{\Delta}_{k-1-j} H\right)\right] \hat{\Delta}_{k-1-i}\left(H \hat{m}_{k-1-i}-z_{k-1-i}\right)
\end{aligned}
$$

where $\prod_{j=0}^{-1}=I d$.

Since $\lim _{k \rightarrow \infty}\left\|\left(F-\Delta\left(V_{\infty}\right) H\right)^{k}\right\|^{1 / k}=\rho\left(F-\Delta\left(V_{\infty}\right) H\right)$ is less than 1 by Lemma 1 , then we have

$$
\begin{aligned}
& \lim _{k \rightarrow \infty}\left\|\hat{m}_{k}-m_{k}^{P}\right\| \\
& \leq\left\|\hat{m}_{k_{1}}-m_{k_{1}}^{P}\right\| \\
& \quad+c_{3} \lim _{k \rightarrow \infty} \sum_{i=0}^{k-k_{1}-1}\left\|\left[\prod_{j=0}^{i-1}\left(F-\hat{\Delta}_{k-1-j} H\right)\right] \Delta_{k-1-i}^{P}\right\| \\
& \quad+\lim _{k \rightarrow \infty} \sum_{i=0}^{k-k_{1}-1} \|\left[\prod_{j=0}^{i-1}\left(F-\hat{\Delta}_{k-1-j} H\right)\right] \hat{\Delta}_{k-1-i}\left(H \hat{m}_{k-1-i}-z_{k}\right.
\end{aligned}
$$

Since by Proposition $1\left\|m_{k}-\hat{m}_{k}\right\|$ is bounded for all $k$, and since $\left\|H m_{k}-z_{k}\right\| \leq c_{2}$, we have that $\left\|H \hat{m}_{k-1-i}-z_{k-1-i}\right\|$ is bounded for all $k$. Thus, using arguments similar to those in the proof of Proposition 1 , we can show that the limits inside $C\left(V_{0}, \hat{V}_{0}, V_{0}^{P}\right)$ exist and are bounded since $\left\|\Delta_{k}^{P}\right\|$ and $\left\|\hat{\Delta}_{k}\right\|$ are bounded.

This means

$$
\begin{aligned}
& \lim _{k \rightarrow \infty}\left\|\hat{m}_{k}-m_{k}^{P}\right\| \leq\left\|\hat{m}_{k_{1}}-m_{k_{1}}^{P}\right\|+C\left(V_{0}, \hat{V}_{0}, V_{0}^{P}\right) . \\
& \text { Since }\left(\mathcal{F}\left(\phi_{k}^{P}, \hat{\phi}_{k}^{E}\right)\right)^{2}=\operatorname{Tr}\left(\hat{V}_{k}\right)+\operatorname{Tr}\left(V_{k}^{P}\right)+\left\|\hat{m}_{k}-m_{k}^{P}\right\|^{2}, \\
& \lim _{k \rightarrow \infty}\left(\mathcal{F}\left(\phi_{k}^{P}, \hat{\phi}_{k}^{E}\right)\right)^{2} \leq \operatorname{Tr}\left(V_{\infty}\right)+\operatorname{Tr}\left(V_{\infty}^{P}\right) \\
&+\left[\left\|\hat{m}_{k_{1}}-m_{k_{1}}^{P}\right\|+C\left(V_{0}, \hat{V}_{0}, V_{0}^{P}\right)\right]^{2} .
\end{aligned}
$$

\section{Proof of Corollary 1}

Statement of Corollary 1: If the distributions of the true evader $\phi_{k}^{E}$, the estimated evader $\hat{\phi}_{k}^{E}$ and the pursuer $\phi_{k}^{P}$ evolve according to the conditions given in Proposition 1 and 2, then $\lim _{k \rightarrow \infty} \mathcal{F}\left(\phi_{k}^{P}, \phi_{k}^{E}\right)$ is bounded.

Proof: Since $\mathcal{F}$ satisfies triangle inequality, we have

$$
\begin{aligned}
& \mathcal{F}\left(\phi_{k}^{P}, \phi_{k}^{E}\right) \leq \mathcal{F}\left(\phi_{k}^{P}, \hat{\phi}_{k}^{E}\right)+\mathcal{F}\left(\hat{\phi}_{k}^{E}, \phi_{k}^{E}\right) \\
& \quad=\mathcal{F}\left(\phi_{k}^{P}, \hat{\phi}_{k}^{E}\right)+\sqrt{\operatorname{Tr}\left(V_{k}\right)+\operatorname{Tr}\left(\hat{V}_{k}\right)+\left\|m_{k}-\hat{m}_{k}\right\|^{2}} .
\end{aligned}
$$

Taking $k \rightarrow \infty$, and using (12) and following, one has

$$
\begin{aligned}
& \lim _{k \rightarrow \infty} \mathcal{F}\left(\phi_{k}^{P}, \phi_{k}^{E}\right) \leq \\
& \lim _{k \rightarrow \infty} \mathcal{F}\left(\phi_{k}^{P}, \hat{\phi}_{k}^{E}\right)+\sqrt{2 \operatorname{Tr}\left(V_{\infty}\right)+\lim _{k \rightarrow \infty}\left\|m_{k}-\hat{m}_{k}\right\|^{2}} \\
& =\lim _{k \rightarrow \infty} \mathcal{F}\left(\phi_{k}^{P}, \hat{\phi}_{k}^{E}\right)+\sqrt{2 \operatorname{Tr}\left(V_{\infty}\right)+\lim _{k \rightarrow \infty}\left(\mathcal{D}\left(\phi_{k}^{E}, \hat{\phi}_{k}^{E}\right)\right)^{2}}
\end{aligned}
$$

Thus by Proposition 1 and $2, \lim _{k \rightarrow \infty} \mathcal{F}\left(\phi_{k}^{P}, \phi_{k}^{E}\right)$ is bounded.

\section{REFERENCES}

[1] Brian D. O. Anderson and John B. Moore. Optimal Filtering (Dover Books on Engineering). Dover Publications, January 2005.

[2] Leonidas J. Guibas, Jean claude Latombe, Steven M. Lavalle, David Lin, and Rajeev Motwani. A visibility-based pursuit-evasion problem. International Journal of Computational Geometry and Applications, 9:471-494, 1996.

[3] R. E. Kalman. A New Approach to Linear Filtering and Prediction Problems. Transactions of the ASME Journal of Basic Engineering, (82 (Series D)):35-45, 1960.

[4] S. M. LaValle. Planning Algorithms. Cambridge University Press, Cambridge, U.K., 2006. Available at http://planning.cs.uiuc.edu/.

[5] S.M. LaValle, D. Lin, L.J. Guibas, and J.C. Latombe. Finding an unpredictable target in a workspace with obstacles. In International Converence on Robotics and Automation (ICRA), volume 1, pages 737-742, April 1997.

[6] S. Lukaszyk. A new concept of probability metric and its applications in approximation of scattered data sets. Computational Mechanics, 33:299-304, 2004. 10.1007/s00466-003-0532-2.

[7] P.E.Caines and D.Q.Mayne. On the discrete time matrix riccati equation of optimal control. International Journal of Control, 12:785794, 1970.

[8] A. Sarmiento, R. Murrieta, and S. A. Hutchinson. An efficient strategy for rapidly finding an object in a polygonal world. In $I N$ PROC. IEEE/RSJ INTERNATIONAL CONFERENCE ON INTELLIGENT ROBOTS AND OPTIMAL SYSTEMS (IROS, pages 1153-1158, 2003.

[9] Sebastian Thrun, Wolfram Burgard, and Dieter Fox. Probabilistic Robotics (Intelligent Robotics and Autonomous Agents). The MIT Press, 2005.

[10] Ren Vidal, Student Member, Omid Shakernia, Student Member, H. Jin Kim, Associate Member, David Hyunchul Shim, and Shankar Sastry. Probabilistic pursuit-evasion games: Theory, implementation and experimental evaluation. IEEE Transactions on Robotics and Automation, 18:662-669, 2002. 